marginal perturbations have directly observable consequences: They affect the non-universal properties of the transition, such as the precise location of the phase boundary as a function of the microscopic parameters (Fig. 1b).

In which quantum systems then could this scenario be actually observed? A natural requirement is a situation in which cooling and $1 / f$ noise balance each other in a way that allows a non-equilibrium steady state to exist. To underpin the basic picture described above, Dalla Torre et al. ${ }^{2}$ elaborate on the case of a resistively shunted noisy Josephson junction. Starting from there, they identify true one-dimensional many-body systems that may exhibit non-equilibrium quantum criticality. They propose studying the effect using either ultracold polar molecules, which have recently become available in the laboratory ${ }^{3,4}$, or trapped ions, which can be controlled with exquisite precision ${ }^{5}$. Chains of molecules or ions may be suitably cooled, while being at the same time subject to fluctuating electric fields as a natural source of $1 / f$ noise. Experimental observation of the fragile quantum critical correlations in these one-dimensional systems will, undoubtedly, be demanding. The challenges will come, for instance, with the required fine tuning of cooling against noise-drive parameters.

One distinguishing aspect of the scenario considered by Dalla Torre and colleagues ${ }^{2}$ is its intrinsic non-equilibrium character. In fact, there are 'smoking gun' signatures for the departure from thermodynamic equilibrium. Possibly the most striking among them is an oscillatory behaviour between energy loss and gain with increasing $1 / f$-noise strength. This implies that the noise may have the effect of a 'pump' to the system, in some analogy to laser driving.

The perhaps counterintuitive fact that noise and dissipation do not necessarily act as an adversary to subtle many-body quantum correlations has been recognized recently in different contexts both theoretically ${ }^{6-8}$ and experimentally $y^{9,10}$, where dissipation is tailored in a way to enforce specific steady states with quantum mechanical properties such as phase coherence in ensembles of bosonic atoms $s^{6}$, or entanglement in spin systems $\mathrm{s}^{7-10}$. The work by Dalla Torre and colleagues adds an intriguing new class of systems to the fledging field of many-body quantum physics beyond thermal equilibrium.

Sebastian Diehl is at the Institute for Quantum Optics and Quantum Information of the Austrian Academy of Sciences, and the Institute for

Theoretical Physics, University of Innsbruck, A-6020 Innsbruck, Austria.

e-mail:Sebastian.Diehl@uibk.ac.at

References

1. Sachdev, S. Quantum Phase Transitions

(Cambridge Univ. Press, 1999).

2. Dalla Torre, E. G., Demler, E., Giamarchi, T. \& Altman, E. Nature Phys. 6, 806-810 (2010)

3. Winkler, K. et al. Phys. Rev. Lett. 98,043201 (2007).

4. Ni, K-K. et al. Science 322, 231-235 (2008).

5. Blatt, R. \& Wineland, D. J. Nature 453, 1008-1015 (2008).

6. Diehl, S. et al. Nature Phys. 4, 878-883 (2008).

7. Verstraete, F., Wolf, M. M. \& Cirac, J. I. Nature Phys. 5, 633-636 (2009).

8. Weimer, H., Müller, M., Lesanovsky, I., Zoller, P. \& Büchler, H. P. Nature Phys. 6, 382-388 (2010).

9. Krauter, H. et al. Preprint at http://arxiv.org/abs/1006.4344 (2010)

10. Barreiro, J. T. et al. Preprint at

http://arxiv.org/abs/1005.1965 (2010).

Published online: 29 August 2010

\title{
ASTRONOMY
}

\section{Galactic left-overs}

This is Messier 63, also known as the sunflower galaxy. First observed in 1779 , by the mid 1800 s its spiral structure had been resolved, making it one of the first spiral galaxies identified. This image - positive at the centre, negative around the edges, and published by David Martínez-Delgado and colleagues in the Astronomical Journal (doi:10.1088/0004-6256/140/4/962; 2010) - shows fresh detail in the galaxy's outermost regions, revealing wispy tendrils that are all that remain of another satellite galaxy, swallowed up by M63.

Similar evidence of galactic guzzling had been seen for the three spiral galaxies that are members of the 'local group' of nearby galaxies (which includes the Milky Way). But Martínez-Delgado et al. have probed deeper into space, building a wider sample of galaxies that are up to 50 million light years away.

Their data support current thinking on galaxy evolution, particularly on the likely chain of events when a dwarf galaxy has the misfortune to get too close to a spiral giant: typically, the uneven gravitational pull of the larger

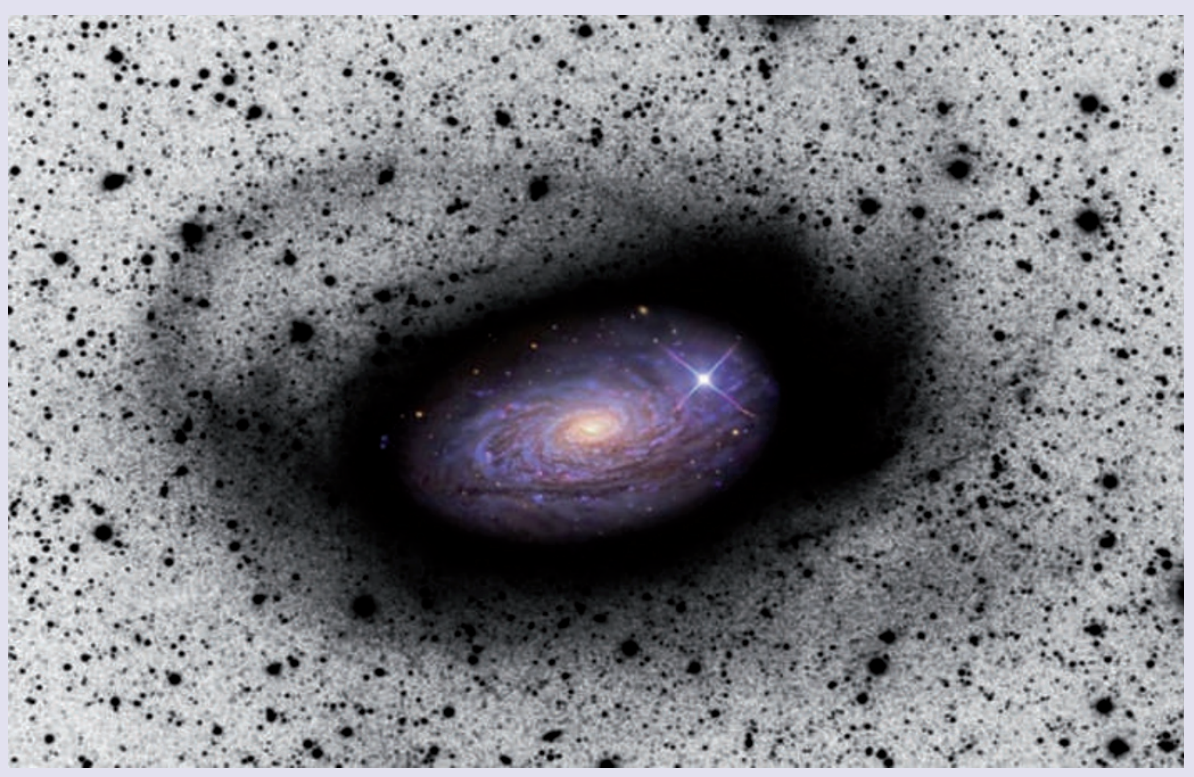

galaxy disrupts the smaller one, dragging its stars into a tidal stream that will eventually - over billions of years - be completely assimilated.

The team collected the data using smallaperture, robotic telescopes at privately run observatories in the USA and Australia, and are already extending their survey to enable more quantitative tests of galaxyevolution models.

ALISON WRIGHT 